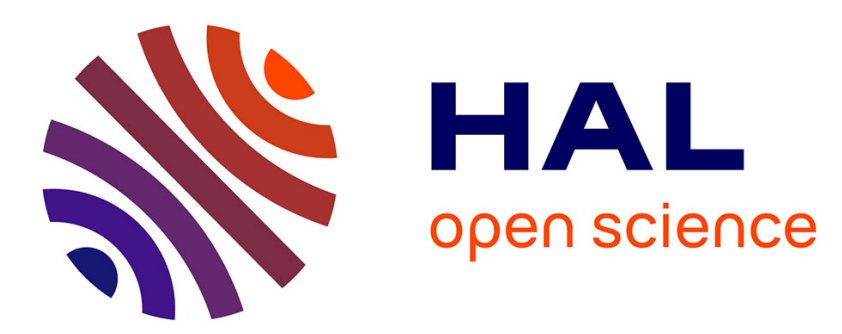

\title{
Dynamical aspects of plasma gratings driven by a static ponderomotive potential
}

\author{
H Peng, C Riconda, M Grech, C-T Zhou, S Weber
}

\section{To cite this version:}

H Peng, C Riconda, M Grech, C-T Zhou, S Weber. Dynamical aspects of plasma gratings driven by a static ponderomotive potential. Plasma Physics and Controlled Fusion, 2020, 62 (11), pp.115015. 10.1088/1361-6587/abb3aa . hal-02993997

\section{HAL Id: hal-02993997 https://hal.science/hal-02993997}

Submitted on 17 Nov 2020

HAL is a multi-disciplinary open access archive for the deposit and dissemination of scientific research documents, whether they are published or not. The documents may come from teaching and research institutions in France or abroad, or from public or private research centers.
L'archive ouverte pluridisciplinaire HAL, est destinée au dépôt et à la diffusion de documents scientifiques de niveau recherche, publiés ou non, émanant des établissements d'enseignement et de recherche français ou étrangers, des laboratoires publics ou privés. 


\title{
Dynamical aspects of plasma gratings driven by a static ponderomotive potential
}

\author{
H. Peng, ${ }^{1,2}$ C. Riconda, ${ }^{2, *}$ M. Grech, ${ }^{2}$ C.-T. Zhou, ${ }^{1, \dagger}$ and S. Weber ${ }^{3,4, \ddagger}$ \\ ${ }^{1}$ Center for Advanced Material Diagnostic Technology, \\ Shenzhen Technology University, Shenzhen 518118, China \\ ${ }^{2}$ LULI, Sorbonne Université, CNRS, École Polytechnique, CEA, F-75252 Paris, France \\ ${ }^{3}$ Institute of Physics of the ASCR, ELI-Beamlines Project, 18221 Prague, Czech Republic \\ ${ }^{4}$ School of Science, Xi'an Jiaotong University, Xi'an 710049, China
}

(Dated: August 14, 2020)

\begin{abstract}
A quasi neutral plasma grating is considered which is generated by two counter-propagating identical laser beams. Typical times scales associated with such grating are given by $t_{\text {unit }}=$ $\sqrt{m_{i} / 2 Z m_{e}}\left(k v_{0}\right)^{-1}$, where $v_{0}$ is the electron quiver in the laser field of wavevector $k$. In most situations the behavior of the grating can be characterized by a single parameter, $\mu$, which is proportional to the ratio of background electron temperature to the square of the electron quiver velocity. Indeed even for quasi-neutral gratings the electron pressure might play an important role, with adiabatic electron heating. The influence on grating formation and lifetime of other parameters that imply the inclusion of dissipative effects or kinetic effects is also examined in detail. Finally, an approximated analytical solution to the fluid model is found that shows good agreement with first-principle simulations.
\end{abstract}

Keywords: plasma grating, laser manipulation, nonlinear plasma dynamics, laser-plasma interaction

\section{INTRODUCTION}

In the last few years there have been worldwide efforts to construct multi-petawatt laser facilities [1-5]. These kinds of power levels prove to be very demanding on optical devices. The intrinsic fluence limitations determine that the optical elements can only sustain ultrashort laser of few hundred $\mathrm{mJ} / \mathrm{cm}^{2}[6-9]$. Thus large-scale optical elements are required which are difficult to manufacture and very expensive.

By contrast, plasmas can withstand very high energy fluxes, up to 6 orders of magnitude higher than solid-state based materials. Also a plasma supports many linear and nonlinear effects which can be employed to control, manipulate and shape ultra-intense laser pulses [10-19]. Plasma optics, although still in its infancy, has therefore attracted a lot of attention in recent years and many successful proof-of-principle experiments have been carried out. A plasma grating is a plasma device that can work as photonic crystal, capable of compressing and stretching chirped pulses, creating optical solitons[20, 21], acting as an optical switch based on the laser frequency or injection angle[19]. A plasma grating can also change the polarization and phase of a laser pulse. They have been used as laser polarizer and waveplate for both, long and moderately intense laser pulses [16-18], as well as short and ultraintense laser pulses [22]. On the surface of a solid target, such a generated grating acts as a plasma hologram for ultraintense lasers [23]. Plasma gratings can also be generated in underdense plasma as volume hologram for laser focusing and mode conversion [24].

\footnotetext{
*Electronic address: caterina.riconda@upmc.fr

${ }^{\dagger}$ Electronic address: zcangtao@sztu.edu.cn

$\ddagger$ Electronic address: Stefan.Weber@eli-beams.eu
}

Moreover, it can be used to couple the laser into surface plasma waves instead of solid grating [25].

Forslund et al. first observed that in the late phase of strongly-coupled stimulated Brillouin scattering, a large amplitude, strongly-modulated and quasi-neutral plasma grating is generated $[26,27]$. The formation of the plasma grating is explained by the steepening of the ion velocity profile and the accumulation of particles in the troughs of the ponderomotive potential created by the beating of counter-propagating laser beams. Its collapse was explained by X-type breaking of the grating [26-28]. Forslund et al. studied the behavior of the ion acoustic waves with initial sinusoidal velocity distribution, and compared the ion kinetic energy with the maximum possible thermal potential, to explain the X-type breaking [29]. However, neither the dynamical effect of the thermal pressure nor the effect of a source term was included. Sheng et al. proposed a linearized fluid model to explain the initial phase of the formation of the plasma grating in the ponderomotive potential of two counter-propagating lasers [30], which quickly fails when the nonlinearity becomes important.

In the present work, we propose a nonlinear model of a plasma grating growing in the static ponderomotive potential generated by two identical counter-propagating lasers. In the considered regime, electrons are localized in the plasma grating and adiabatic electron heating should be considered. This configuration and the model we propose is adapted to give correct predictions for pulses on the picosecond/femtosecond scale with relatively high intensity. These high intensities allow to reach high peak values of the grating close to or even higher than $n_{c}$, necessary for many applications. The ratio of the adiabatic thermal potential to the ponderomotive potential (denoted by $\mu$ ) is crucial to determine the dynamics of the plasma grating[31]. In this paper, the details of the growth, transient state and collapse are considered us- 
ing both fluid and PIC (particle-in-cell) simulations, a detailed comparison is also performed. Good agreement is found between the two methods within the limits of the fluid approximation. Particular emphasis are put on investigating kinetic effects with PIC simulations in the long timescales, which is beyond the limits of the fluid approximation. The role of parameters affecting the dynamics of the gratings such as plasma density, ion temperature and particle collisions are also discussed.

This paper is organized as follows: in Sec.II, the equations describing the model are presented. Sec.II A presents the macroscopic approach and the following section, Sec.IIB, compares the results of macroscopic and microscopic simulation. In Sec.III, depending on the value of a characteristic parameter, $\mu$, three regimes are found, in which the plasma grating displays very different behavior: complete reflection, partially reflecting and partially crossing, and crossing. In Sec.IV, the various time scales of importance to the dynamics are briefly discussed. The following section, Sec.V, it is discussed how the use of the interaction parameters allow to customize gratings for experiments. Sec.VI discusses how kinetic effects such as a finite ion temperature and particle collisions affect the grating dynamics. Finally, in Sec.VII, conclusions are presented.

\section{MODELLING THE GRATING DYNAMICS}

The details of the growth, transient state and collapse are considered using both fluid and PIC (particle-in-cell) simulations, which describe the grating dynamics from the macroscopic and the microscopic point of view, respectively.

As is shown subsequently, the two methods agree very well as far as the essential aspects of grating formation are concerned. The macroscopic fluid approach is more intuitive and allows better to understand simple scaling and establish governing parameters. The kinetic (PIC) approach is a first-principle-approach which includes by definition all physically relevant processes, in particular such processes as wavebreaking which can not be described in the fluid picture. The kinetic approach therefore allows to follow the dynamics all the way.

As far as a full understanding is concerned the two approaches can be considered complementarily and are both needed for a full understanding. In the following the macroscopic equations are presented in Sec.II A and the comparison with kinetic simulations is presented in Sec.II B.

\section{A. Governing macroscopic equations}

The nonlinear two-fluid equations including the ponderomotive potential and neglecting the electron momen- tum are:

$$
\begin{gathered}
0=e \frac{\partial \phi}{\partial x}-\frac{1}{n_{e}} \frac{\partial p}{\partial x}-e \frac{\partial \phi_{p}}{\partial x} \\
\frac{\partial^{2} \phi}{\partial x^{2}}=4 \pi e\left(Z n_{e}-n_{i}\right) \\
\frac{\partial n_{i}}{\partial t}+\frac{\partial\left(n_{i} v_{i}\right)}{\partial x}=0 \\
\frac{\partial v_{i}}{\partial t}+v_{i} \frac{\partial v_{i}}{\partial x}=-\frac{Z e}{m_{i}} \frac{\partial \phi}{\partial x}
\end{gathered}
$$

The ponderomotive potential $\phi_{p}=\frac{1}{2} \frac{m_{e} c^{2}}{e} a_{0}^{2} \cos (2 k x)$ is generated by two identical counter-propagating lasers in the plasma. Here $k$ is the laser wave vector in the plasma and $a_{0}$ is the amplitude of lasers. $k=$ $k_{0} \sqrt{1-n_{0} / n_{c}}$, where $k_{0}, n_{0}$ are the laser wavevector in vacuum and unperturbed plasma density, respectively. Adiabatic heating is considered. Thus:

$$
\begin{gathered}
p n_{e}^{-\gamma}=\text { const } \\
p=n_{e} T_{e} .
\end{gathered}
$$

$\gamma=(2+D) / D, D$ are the adiabatic index and number of freedom, respectively. The grating is one-dimensional, so $D=1$ and $\gamma=3$. Then the thermal potential is $\phi_{t h}=$ $\frac{3}{2} \frac{T_{0}}{e}\left(\frac{n_{e}}{n_{0}}\right)^{2}$, where $T_{0}$ is the initial electron temperature.

Then Eq(1a) leads to:

$$
\phi=\phi_{p}+\phi_{t h}
$$

The ponderomotive force is $f_{p}=-e \partial \phi_{p} / \partial x$. One can further normalized these equations with:

$$
\begin{gathered}
x_{\text {unit }}=\frac{1}{2 k}, \quad t_{\text {unit }}=\sqrt{\frac{1}{2} \frac{m_{i}}{Z m_{e}}}\left(k v_{0}\right)^{-1}, \\
v_{\text {unit }}=\sqrt{\frac{1}{2} \frac{Z m_{e}}{m_{i}}} v_{0}, \quad n_{\text {unit }}=n_{0} .
\end{gathered}
$$

Then $\mathrm{Eq}(1)$ can be transformed to:

$$
\begin{gathered}
\mu \frac{\partial^{2} n_{e}^{2}}{\partial x^{2}}-\cos x=\nu\left(Z n_{e}-n_{i}\right), \\
\frac{\partial n_{i}}{\partial t}+\frac{\partial\left(n_{i} v_{i}\right)}{\partial x}=0 \\
\frac{\partial v_{i}}{\partial t}+v_{i} \frac{\partial v_{i}}{\partial x}=\sin x-\mu \frac{\partial n_{e}^{2}}{\partial x}
\end{gathered}
$$

where $\mu=3 T_{0} / m_{e} v_{0}^{2}, \nu=\left(\omega_{p 0} / \sqrt{2} k v_{0}\right)^{2}, v_{0}$ is the electron oscillation velocity for nonrelativistic laser intensities and $\omega_{p 0}$ is the initial electron plasma frequency. Note that $\nu<1$ is used as a criterion of the superradiant regime[32], where the electron dynamics are very important and the electron momentum cannot be neglected. In this regime, the grating dynamics are much faster(usually on the order of tens of femtosecond). While we are focusing on ion-dominant plasma grating which is slowly evolving and quasistationary. This kind of plasma grating is suitable for manipulation of laser pulses with duration going up to $100 \mathrm{fs}$ or even few ps. So this regime $(\nu<$ 
1) should be avoided in the discussion here. Note also that the strong coupling stimulated Brillouin scattering takes place when $\left(v_{0} / v_{t h}\right)^{2}>4 k_{0} c_{s} \omega_{p} / \omega_{p 0}^{2}[33]$, which corresponds to $\mu<\mu_{t h}=\left[2 a_{0}^{2} \frac{m_{e}}{m_{i}}\left(\frac{n_{c}}{n_{0}}\right)^{2}\left(1-\frac{n_{0}}{n_{c}}\right)\right]^{-1 / 3}$. One finds that in the case $\nu \gg 1, \operatorname{Eq}(5 \mathrm{a})$ can be simply replaced with $Z n_{e}=n_{i}$.
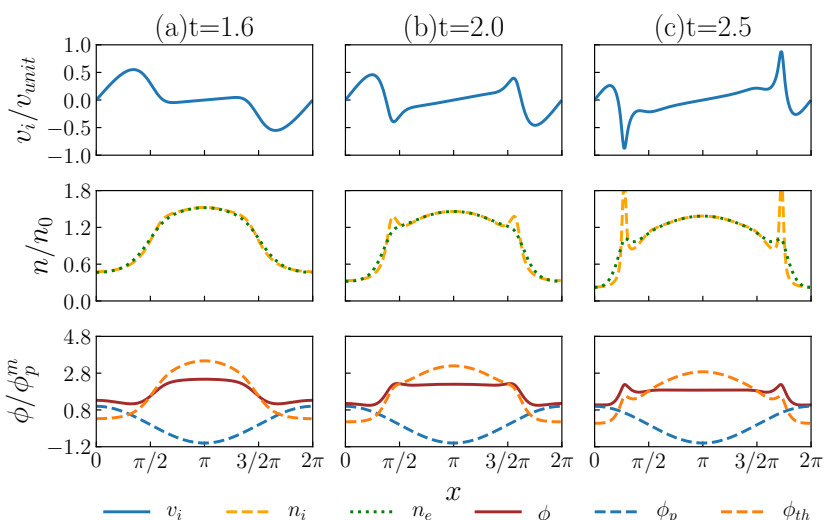

FIG. 1: Simulation results of the fluid model with $\mu=1.5$ and $\nu=59.5$. Shown are ion velocity, plasma density, thermal potential, ponderomotive potential and total potential at different times, respectively. All the potentials are normalized to the maximum ponderomotive potential: $\phi_{p}^{m}=m_{e} / 2 e v_{0}^{2}$.

\section{B. Macroscopic and microscopic comparison of the process}

To validate the fluid model formulated above, we first numerically solve it and compare the results to two PIC simulations with the same $\mu$ and $\nu$ : $\mu=1.5$, $\nu=59.5(Z=1$ is used throughout the paper unless specified). The simulation results of the fluid model are shown in Fig(1). Because the ponderomotive potential is static and periodic along the $\mathrm{x}$ axis, only one period is shown and periodic boundary conditions are applied. There are obviously three different stages of the plasma grating, which are shown in (a), (b), (c), respectively.

At first, the ion velocity grows and steepens under the drive of the ponderomotive potential, leading to the gathering of ions and electrons towards the potential trough. But the thermal potential $\phi_{t h}=\frac{3}{2} \frac{T_{0}}{e}\left(\frac{n_{e}}{n_{0}}\right)^{2}$ also grows quickly as the electron density grows quickly in the grating. The combined potential of $\phi_{p}$ and $\phi_{t h}$ has a plateau, which then stop the ions moving towards the grating. A velocity plateau is established.

Then in the second stage, as the ion kinetic energy is not big enough for the ions to climb up the potential barrier and pass it $P<e \Delta \phi, P$ is the kinetic energy of the ions), the ions keep accumulating at each edge of the grating. And the grating grows "two spikes" of ions. The following ions are completely reflected, leading to a $\mathrm{X}$-like ion phase space that can only be observed in PIC simulations(see following discussion on PIC simulation results). This corresponds to the X-type breaking that has been widely discussed[27-29, 34].

In the last stage, the reflected ions lead the grating to expand. The ions co-propagating with the edges of the potential barrier are accelerated to high velocity. The plasma density in the grating starts to decrease as the grating gets stretched. As more and more ions accumulates at each edge, the ion density is deviated from the electron density significantly at each edge.
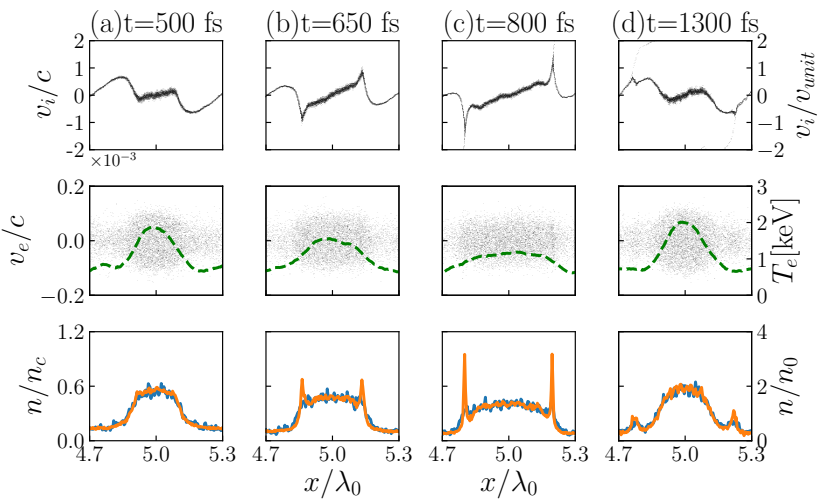

FIG. 2: The results of the first PIC simulation at different times. Note that Fig(a),(b),(c) are at the same normalized times as Fig(a),(b),(c) of Fig(1), respectively. $t_{\text {unit }} \simeq 320 \mathrm{fs}$ here. From upper row to lower row are the ion phase space, electron phase space and local electron temperature(green line, ticked on the right), ion density(red line) and electron density (blue line) at different times in the center of the simulation box, respectively. The ion velocity and plasma density in normalized units are ticked in the right. Note that a SavitzkyGolay filter is applied to smooth the electron temperature to increase the precision without distorting the trend.

The temporal evolution of the plasma gratings in the center of the simulation box obtained from the PIC simulations are shown in $\operatorname{Fig}(2)$ and Fig(3). In the first PIC simulation showing in $\operatorname{Fig}(2)$, the laser wavelength, laser amplitudes, electron temperature, unperturbed plasma density are $\lambda_{0}=1 \mu \mathrm{m}, I=5 \times 10^{15} \mathrm{~W} / \mathrm{cm}^{2}\left(a_{0} \simeq 0.06\right)$, $T_{e}=0.92 \mathrm{keV}, n_{0}=0.3 n_{c}$, respectively. In the second PIC simulation showing in Fig(3), the laser wavelength, laser amplitudes, electron temperature, unperturbed plasma density are $\lambda_{0}=1 \mu \mathrm{m}, I=1 \times 10^{16} \mathrm{~W} / \mathrm{cm}^{2}\left(a_{0} \simeq\right.$ $0.0855), T_{e}=1.84 \mathrm{keV}, n_{0}=0.46 n_{c}$, respectively. In both PIC simulations, $\mu=1.5$ and $\nu=59.5$. All the lasers have slowly linearly growing fronts of $10 \pi \omega_{p 0}^{-1}$ to prevent the triggering of the Langmuir oscillation of the electrons and the remainder of the laser pulse is constant. Plasma densities above quarter-critical are used throughout the paper, in order to avoid the noise-induced Raman scattering and the interference of the resultant Raman heating. The ion temperatures in both PIC simulations are $T_{i}=1 \mathrm{eV}$. Note that here the strong coupling threshold is $\mu_{t h} \simeq 32$. Thus we are in the strong coupling regime, which means at the beginning the thermal potential is much smaller than the ponderomotive potential 
and can be neglected. The one-dimensional SMILEI code [35] is used for the PIC simulations. Cell size of $\lambda_{0} / 256$, 50 particles per cell, $m_{i} / m_{e}=1836$ and $Z=1$ are used. We use a $6 \lambda_{0}$ plasma plateau with $2 \lambda_{0}$ vacuum at each side of the simulation boxes.

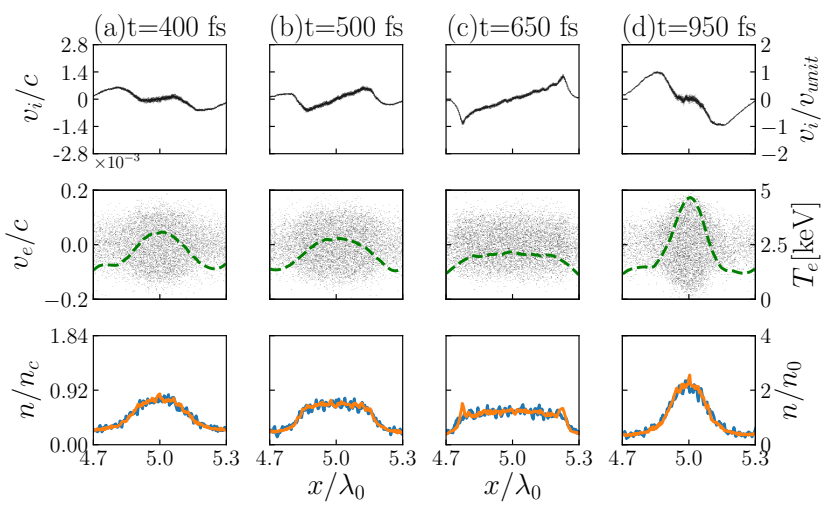

FIG. 3: The results of the second PIC simulation at different times. Note that Fig(a),(b),(c) are at the same normalized times as $\operatorname{Fig}(\mathrm{a}),(\mathrm{b}),(\mathrm{c})$ of $\operatorname{Fig}(1)$, respectively. $t_{\text {unit }} \simeq 256 \mathrm{fs}$ here. The layout is the same as that of $\operatorname{Fig}(2)$.

The same three stages are found easily in the PIC simulations. They agree well with the fluid simulation results. The electron temperature in the grating does increase as the electrons are compressed in the grating from the initial $920 \mathrm{eV}$ to about $2 \mathrm{keV}$ in $\mathrm{Fig}(2)$, from $1.84 \mathrm{keV}$ to $3.08 \mathrm{keV}$ in $\mathrm{Fig}(3)$, and decrease as the grating gets stretched. Fig(d) of both figures show the start of another round of formation of plasma grating. The fluid simulation fails at this time, because that the ion velocity is double-valued in the spikes. The ion spikes collide with the counter-propagating ones and coalesce into a bigger one and separate from each other after that. The plasma gratings are compressed to a higher amplitude in the second round. The possible reason is that in the first round, the lasers have a slow rising front but not in the following rounds. During this whole process, compression and stretch of the plasma, a small part of ions are ejected to each direction.

Notice that even if the inital laser parameters are the same in the PIC and fluid simulation, we expect a small systematic discrepancy in the grating parameters, due to the following effect : once the plasma gratings are established, the grating's dispersion starts to have a band gap structure[19, 20, 36], and the driving lasers get reflected, which is not included in fluid simulation. This effect is discussed in detail in Appendix(A). Note that it is applicable here to neglect the energy exchange between the lasers, because that the growth length of the strong coupling $c / \gamma_{s c}$ (about $24.4 \mu \mathrm{m}$ and $18 \mu \mathrm{m}$ in Fig(2) and Fig(3) respectively) is much larger than the plasma length. The consistency between the fluid simulation and PIC simulation approves our theoretical model and it shows that indeed the electrons are adiabatically com- pressed in the grating, $\mu$ and $\nu$ are a pair of important parameters to determine the grating behavior. The analytical solution of the fluid model in a simplified case is discussed in Appendix(B).

\section{THE ROLE OF THE PARAMETER $\mu$ AS FAR AS THE ION DYNAMICS IS CONCERNED}

Apparently the second stage, i.e. the stage when the velocity plateau forms, is crucial to determine the subsequent behavior of the grating. In Fig(4), velocity plateaus with different $\mu$ are shown. At the time of this transient stage $\left(t=t_{\text {plateau }}\right)$, one can compare the maximum kinetic energy of the ions $P_{m}$ with the amplitude of the potential barrier $e \Delta \phi=e \phi_{\max }-e \phi\left(P_{m}\right)\left(\phi_{\max }\right.$ is the maximum total potential and $\phi\left(P_{m}\right)$ is obtained at the $x$ where ions have the peak velocity) to know how the ions act: if $P_{m}<e \Delta \phi$, the ions will be reflected and the grating expand, as the case shown above; if $P_{m}>e \Delta \phi$, then the ions cross the potential barrier. As shown in the simulation, with a big $\mu($ e.g. $\mu=1.5), P_{m}-e \Delta \phi$ is negative. And obviously in the cold $\operatorname{limit}(\mu=0), P_{m}-e \Delta \phi$ is positive.
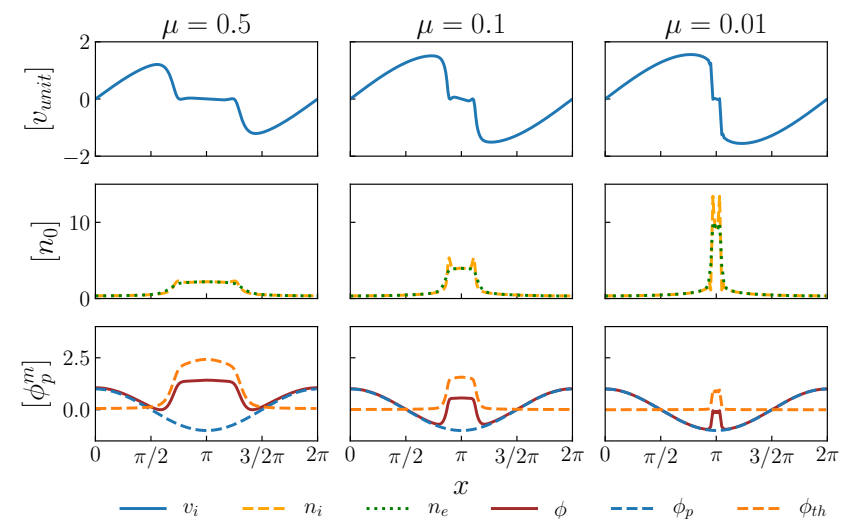

FIG. 4: Fluid simulation of the second stages with different $\mu$. $\nu$ is fixed at 59.5. Normalized units are used.

Fig(4) can be explained as follows: as $\mu$ decreases, the thermal potential $\phi_{t h}=\mu\left(n_{e}\right)^{2}$ in the grating needs higher grating density to stop the ions moving towards the grating. Higher grating density is obtained closer to the center as the wave steepens. Thus the grating has smaller width when the velocity plateau forms(note that the grating width and amplitude are defined as the width and electron density of the density plateau, respectively). But the increase of the grating density doesn't compensate the decrease of $\mu$, so the potential barrier $e \Delta \phi$ decreases. One the other side, the ions can get closer to the center and are accelerated to higher kinetic energy. One can expect that at a certain $\mu, P_{m}-e \Delta \phi$ flips its sign from negative to positive and the ions starts to pass through the potential barrier. Finally, at a very small $\mu$, the bulk of the ions can pass through the potential 
barrier. The grating is in the crossing regime.
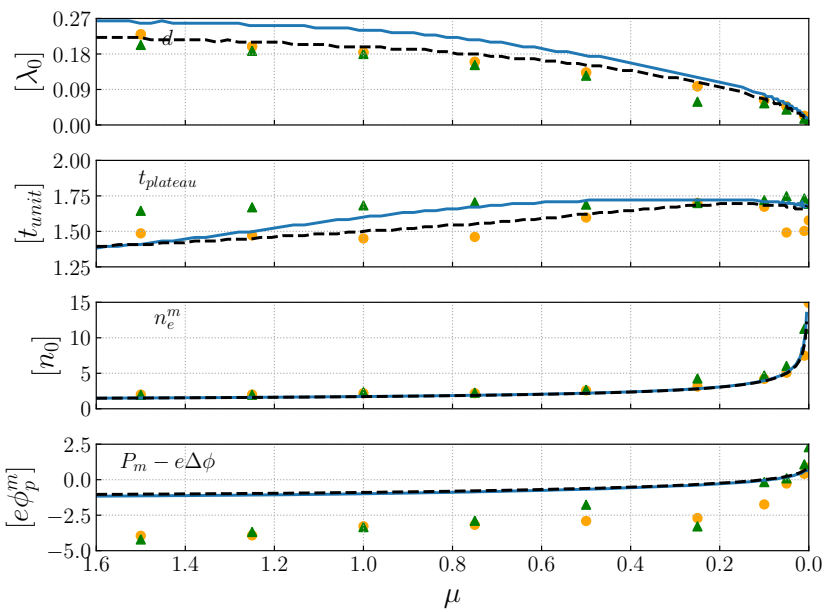

FIG. 5: From top to bottom are: the minimum grating width $d$, the time to form the velocity plateau $t_{\text {plateau }}$, the maximum electron density $n_{e}^{m}, P_{m}-e \Delta \phi$ (normalized by the maximum ponderomotive potential $\left.e \phi_{p}^{m}=m_{e} v_{0}^{2} / 2\right)$ at $t_{\text {plateau }}$, respectively. Fluid simulation results are shown with blue $\operatorname{lines}(\nu=59.5)$ and black broken line $(\nu=19.5)$. The PIC simulation with varying temperature are shown with green triangles $\left(I=5 \times 10^{15} \mathrm{~W} / \mathrm{cm}^{2}, \nu=59.5\right)$ and orange circles $\left(1.5 \times 10^{16} \mathrm{~W} / \mathrm{cm}^{2}, \nu=19.5\right)$, both with $n_{0}=0.3 n_{c}$ and other parameters the same as those of $\operatorname{Fig}(2)$.

In both the reflection case and the crossing case, the grating starts to expand after the second stage. Thus the minimum grating width $d$, maximum grating amplitude (expressed by $\left.n_{e}^{m}\right)$ is obtained at this time $\left(t=t_{\text {plateau }}\right)$. How these parameters and $P_{m}-e \Delta \phi$ evolve with $\mu$ is shown in Fig(5) with both fluid simulation results and PIC simulation results. Fluid simulations agree with the PIC simulations quite well.

In the fluid simulations, at $\mu=0.1(\nu=95.5)$ and $\mu=0.085(\nu=19.5), P_{m}-e \Delta \phi$ flips its sign. In PIC simulation, complete reflection is found at $\mu>0.25$. But for $0.25>\mu>0.001$, a transition regime where the ions are firstly reflected then crossing is found, which is discussed in details in the next section. For $\mu<0.001$, we see directly crossing. Fluid simulations fail to describe the reflection and crossing of the ions in the transition regime and crossing regime because the fluid simulation doesn't allow the variables to be double-valued. The large deviation of negative $P_{m}-e \Delta \phi$ between the fluid simulations and the PIC simulations can be observed in the last figure of $\mathrm{Fig}(5)$. It can be explained by the fact that slowly-growing front is applied to the lasers in PIC simulations, which leads to a smaller maximum ion velocity thus a smaller maximum ion kinetic energy $P_{m}$ in PIC simulations.

In the case of $\nu \gg 1$, it is found that $\nu$ has little effect on the main results obtained above. This can be readily known from $\mathrm{Eq}(5 \mathrm{a})$ and from the simulation results with different $\nu$ in $\operatorname{Fig}(5)$. But note the value of $\nu$ af-

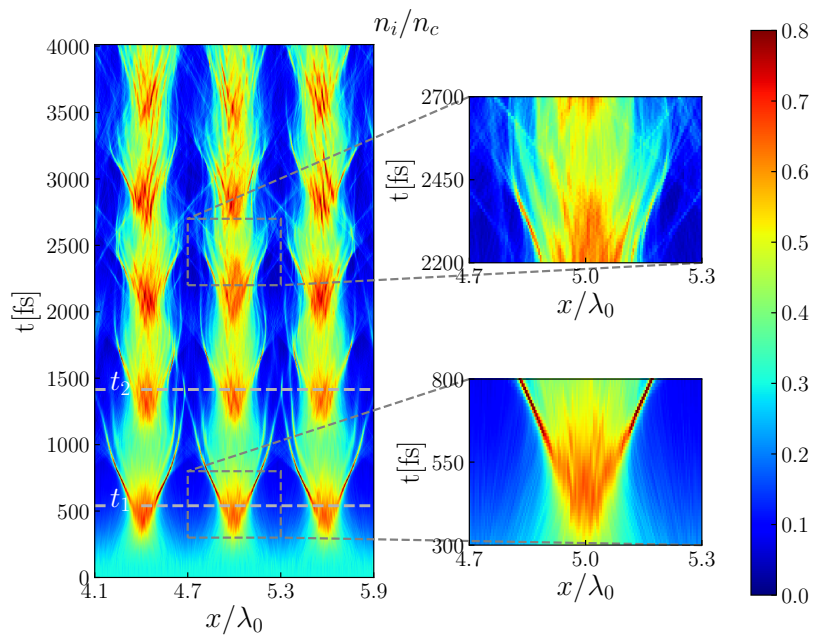

FIG. 6: Ion density evolution in time at the center of the simulation box. The periodic formation and stretching of the plasma grating at $\mu=1.0$ is seen. The two insets on the right show the zooming in of the main figure at different times. Note that $t_{1}=540 \mathrm{fs}, t_{2}=1415 \mathrm{fs}$.

fects the amplitude of the ion spikes at the edges of the grating in the complete reflecting regime. With smaller $\nu$ (still much larger than 1), the spike amplitude is larger and vice versa. But when $\nu$ is close to 1 , the electron dynamics become important and can no longer be neglected, which has been proven by simulations using cold nonlinear two-fluid equations and PIC simulation (not shown). The case of $\nu<1$, as mentioned above, corresponds to the superradiant regime[32]. The electrons form a grating in a very short timescale, during which the ions barely move. But this paper is devoted to study the plasma grating with long time scale, which is suitable to manipulate femtosecond laser pulses. The case $\nu<1$ is beyond this paper's scope and left out of discussion.

\section{CHARACTERISTIC TIME SCALES OF THE NONLINEAR GRATING DYNAMICS}

PIC simulations are conducted to show long-term plasma density evolution in time for different $\mu$. For this section the parameters of the PIC simulations, except the temperature, are the same as in $\mathrm{Fig}(2)$. For relatively large $\mu(\mu \geq 0.25)$, the grating forms and expands, the ions concentrate and get reflected periodically, as shown in Fig(6), in which $\mu=1$. The periodicity time $T_{\text {grating }}$ is roughly inversely proportional to $\mu$, as is shown in $\operatorname{Fig}(11)$. As seen in $\operatorname{Fig}(6) T_{\text {grating }}=t_{2}-t_{1}\left(t_{1}\right.$ is the establish time of the grating, note that $t_{1}=t_{\text {plateau }} . t_{2}$ is the time that the grating stretches to its largest extent, respectively) is very regular and sustained over many periods. However, this changes if $\mu$ is decreased as the periodicity becomes irregular as discussed below. If $\mu$ is 


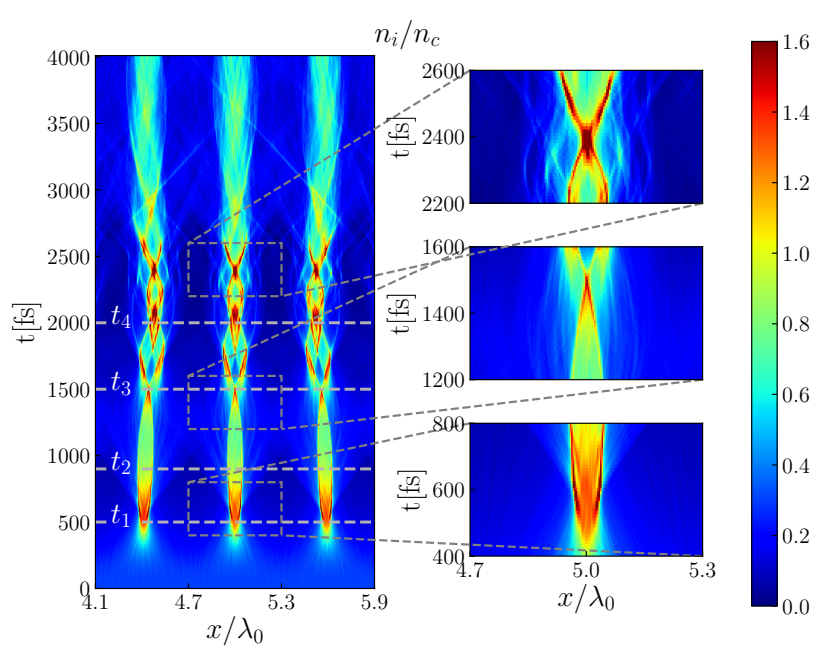

FIG. 7: Ion density evolution in time for $\mu=0.1$. The three insets on the right show the zooming in of the main figure at different times. Note that $t_{1}=500 \mathrm{fs}, t_{2}=900 \mathrm{fs}, t_{3}=1500 \mathrm{fs}$, $t_{4}=2000 \mathrm{fs}$.
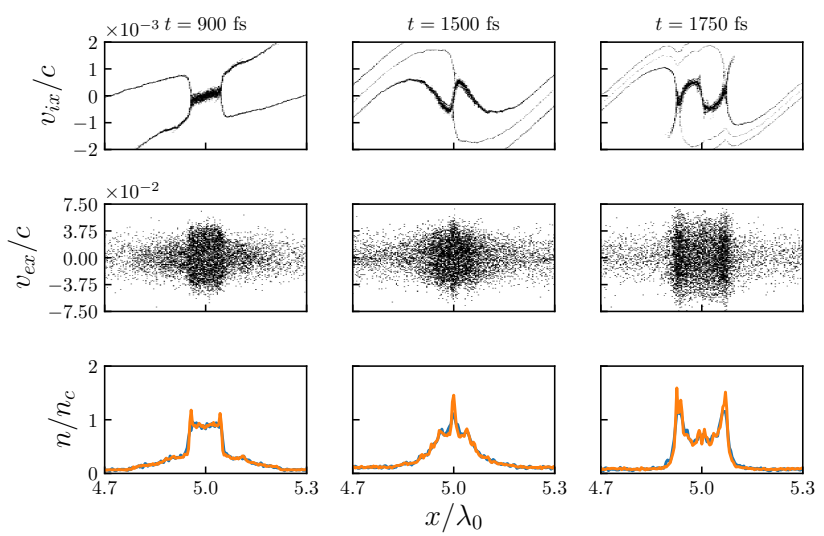

FIG. 8: Shown are the ion phase space, electron phase space and plasma density at different times when $\mu=0.1$.

smaller than 0.25 , one has the transition regime. The dynamics of the electrons and ions are more complicated, e.g. see $\operatorname{Fig}(7)$ in which $\mu=0.1$. The ions are pushed to the center to form the velocity plateau at $t_{1}$. Then the ions are firstly reflected because of $P_{m}<e \Delta \phi$, the grating expands and the grating amplitude gets smaller, so do $\phi_{t h}$ and $\Delta \phi$. At $t_{2}, P_{m}-e \Delta \phi$ flips its sign to positive, the ions with the highest kinetic energy now are able to climb up the potential barrier in the center instead of being reflected. Then the grating shrinks again. But still a big portion of ions are reflected as shown in Fig(8). At $t_{3}$, the ions meet in the center and a very narrow grating is formed. This time, the ions have larger kinetic energy and the grating amplitude is smaller. The bulk of the ions have the kinetic energy $P>e \Delta \phi^{\prime}$ and are able to traverse the center and cross each other. But they are

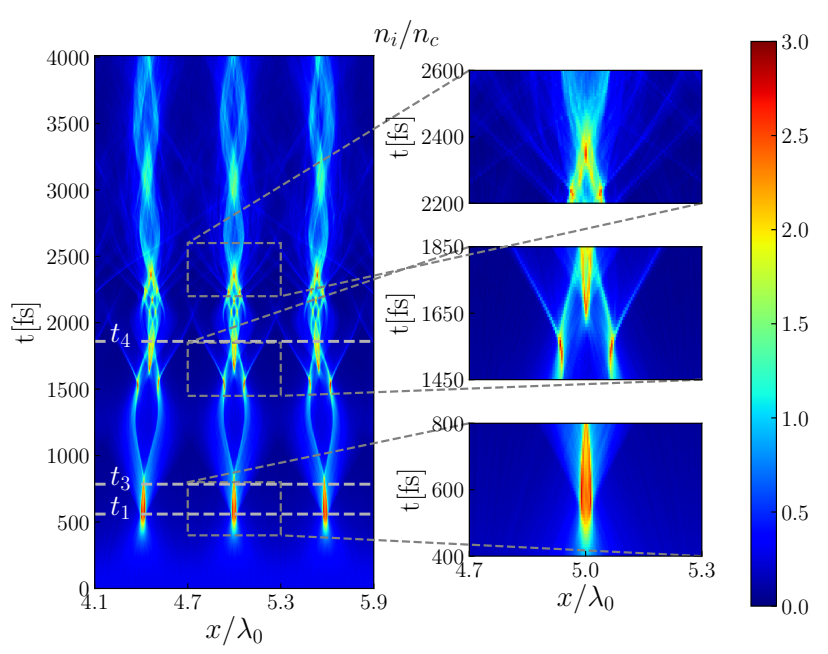

FIG. 9: Ion density evolution in time at $\mu=0.02$. The three insets on the right show the zooming in of the main figure at different times. Note that $t_{1}=560 \mathrm{fs}, t_{3}=785 \mathrm{fs}, t_{4}=1860 \mathrm{fs}$.

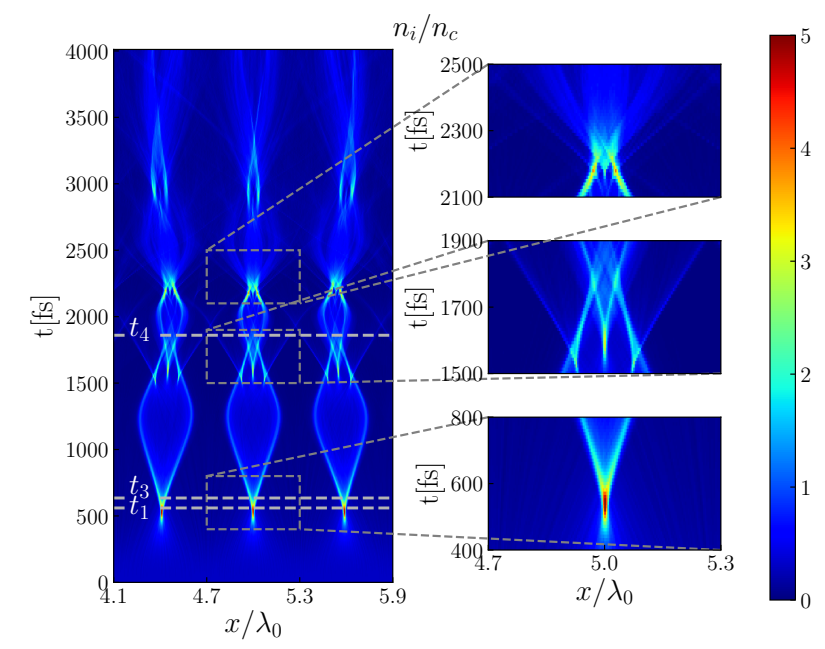

FIG. 10: Ion density evolution in time at $\mu=0.0$. The three insets on the right show the zooming in of the main figure at different times. Note that $t_{1}=560 \mathrm{fs}, t_{3}=635 \mathrm{fs}, t_{4}=1860 \mathrm{fs}$.

dragged back by the ponderomotive potential, and cross the center at $t_{4}$ again. After two more rounds of oscillation of particles in the ponderomotive potential, the particles are phase-mixed and no more gratings occur. The expansion of the grating due to the reflection, the subsequent shrinking and crossing at $t=900 \mathrm{fs}, 1500 \mathrm{fs}$, 1750 fs are shown in Fig(8).

The ion density evolution in time at $\mu=0.02$ and $\mu=0$ are shown in Fig(9) and Fig(10). For smaller $\mu$, except that at the time the velocity plateau forms, $t_{\text {plateau }}$, the grating width is smaller and the grating amplitude is larger, the life time of the grating, $T_{\text {life }}=t_{3}-t_{1}$, is 

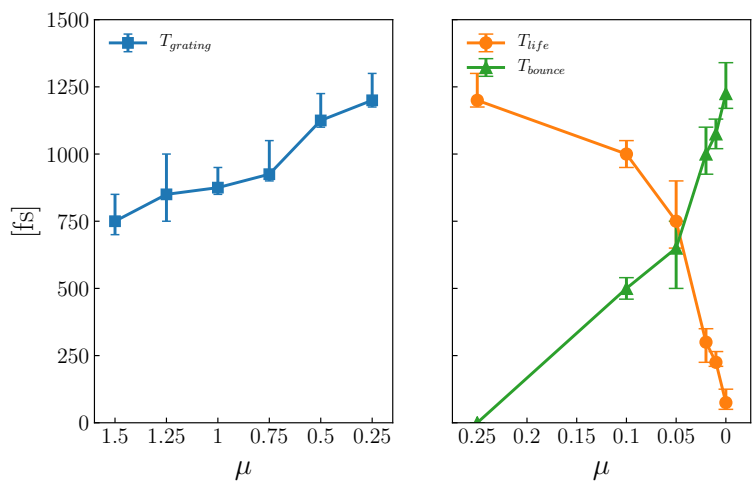

FIG. 11: Left: the time period of grating formation and collapse $T_{\text {grating }}$ for relatively big $\mu$. Right: the life time of the grating $T_{\text {life }}$ and the following bouncing time of ions in the ponderomotive potential $T_{\text {bounce }}$ for relatively small $\mu$.

shorter, the time of crossing and being dragged back by the ponderomotive force $\left(T_{\text {bounce }}=t_{4}-t_{3}\right)$ is longer, as shown in $\operatorname{Fig}(11)$. At $\mu=0$, the grating only exists for a very short time and then dies out quickly.

\section{PARAMETER WINDOW FOR EXPERIMENTS}

The analysis of the fluid equations and the PIC simulations allows to identify two values of the $\mu$ parameter that correspond to a transition between different regimes. The threshold value below which the system goes from complete reflection to partial reflection (transition regime) is $\mu=0.25$. As $\mu$ decreases and reaches the threshold value $\mu=0.001$, the system enter the crossing regime. Another threshold $\mu=2$ is identified in PIC simulation (not shown), above which the grating is in the low-intensity regime, which means that the laser intensity is not high enough to produce a functional plasma grating. In this regime, as $\mu$ increases, the system slowly transitions to the weakly-coupled SBS. These three lines and the relation between $\mu$ and laser intensity $I$ and $T_{e}$ are shown in Fig(12).

In the same figure we superimpose some dots that corresponds to parameters taken from the simulations in Refs[19] and [36] for comparison. For a given value of $\mu$, and roughly independent on $\nu$ [31] the characteristics of the plasma gratings are fixed, and in particular the amplitude of the plasma grating is inversely proportional to $\mu$. Thus for a given laser amplitude $a_{0}=0.05$ increasing the temperature corresponds to increasing $\mu$ and thus to obtain smaller plasma grating. Analogously, for a given initial electron temperature $T_{0}$ fixed at $25 \mathrm{eV}$, increasing the laser amplitude corresponds to decreasing $\mu$ and obtaining plasma gratings at higher amplitude. This is exactly what it is obtained in the aforementioned papers. The orange circle and the cross in the Fig(12) have very close values of $\mu$ (equal to 0.163 and 0.176 , respectively), and the corresponding gratings in PIC simulation have very similar dynamics (as shown in Fig(4) of Ref[19]). This also happens to the upward triangle and the plus sign $(\mu=0.09$ and 0.12$)$, the rightward triangle and the leftward triangle $(\mu=0.024$ and 0.04$)$.

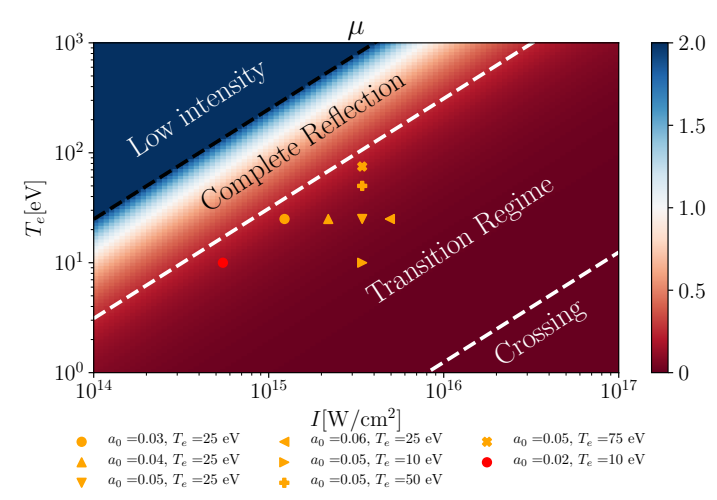

FIG. 12: The relation between $\mu$ and laser intensity $I$ and $T_{e}$. The upper broken line: $\mu=2$. The middle broken line: $\mu=0.25$. The lower broken line: $\mu=0.001$. The orange dots and red dot are from G. Lehmann's simulation[36] and [19], respectively.

The result from Ref[36] (red dot) cannot be compared directly with the simulations in Ref[19] even if the value of $\mu$ is the same since in those simulations the pump lasers have finite durations and the driving ponderomotive potential exists for only $250 \mathrm{fs}$. As a consequence, the grating are not driven to grow to the maximum amplitude they can attain. In this paper we solve the model equations for a long-standing ponderomotive potential, and the results are summarized in $\operatorname{Fig}(5)$. We can thus predict the peak grating amplitude and formation time in Ref[19], while the grating amplitude in Ref[36] is overestimated. However the fluid model is still valid, so to obtain the value of the density grating in a general situation, it is enough to solve the model equation with a ponderomotive driver of finite duration, or as an initial condition problem. PIC simulations are necessary to obtain the subsequent evolution of the grating, however as we have shown we can identify typical behaviours associated to a given value of $\mu$. This allows in a straightforward way to identify the plasma and laser conditions necessary in order to produce experimentally a specific grating. In the following, we examine how other parameters can also affect the the grating formation and should be considered in some particular cases. 


\section{THE ROLE OF KINETIC EFFECTS FOR THE GRATING GENERATION}

\section{A. Influence of a finite ion temperature}

For very low ion temperature, that is when the velocity spread in the ion phase space $\delta v=\sqrt{T_{i 0} / m_{i}}$ is much smaller than the maximum ions velocity in the ponderomotive potential $v_{m}: \delta v \ll v_{m}$, the fluid model proposed so far gives the correct description of the system. We can deduce the condition of validity of the fluid model by considering that the maximum kinetic energy of the ions $P_{m}=m_{i} v_{m}^{2} / 2$ is smaller but on the same order of the maximum ponderomotive potential $\left(e \phi_{p}^{m}=m_{e} v_{0}^{2} / 2\right)$. Then the inequality above becomes $\sqrt{T_{i 0} / m_{e} c^{2}} \ll a_{0}$, usually satisfied in experiments. This is verified by PIC simulations with $T_{i}=50 \mathrm{eV}$. The other parameters are the same as in $\operatorname{Fig}(2)$, and in particular $a_{0}=0.06$, so that $e \phi_{p}^{m}=0.92 \mathrm{keV}$. The results are shown in Fig(13). The plasma grating has the same behaviour as before, only in this simulations the ion spikes at the edges of the grating are smoothed out.
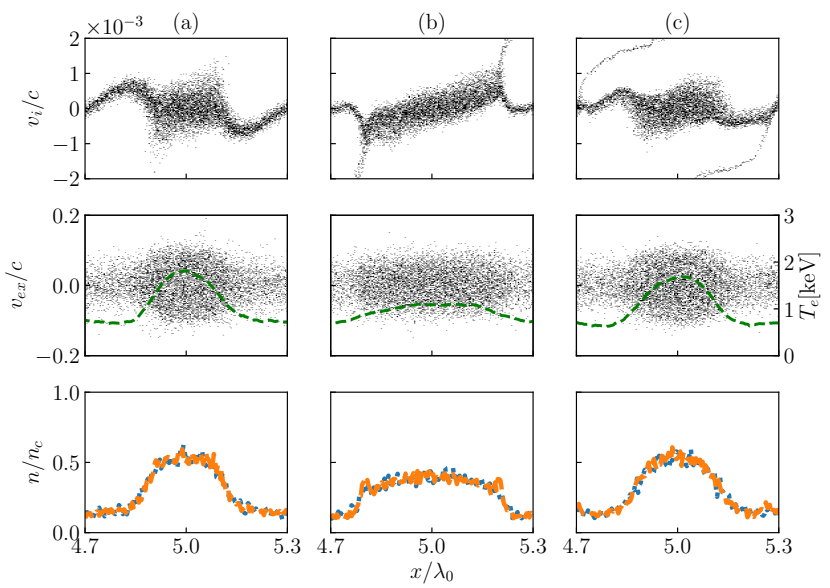

FIG. 13: PIC simulation with $T_{i}=50 \mathrm{eV}$, others are the same as $\operatorname{Fig}(2) . \quad t=500 \mathrm{fs}, 800 \mathrm{fs}, 1300 \mathrm{fs}$ in (a), (b), (c), respectively.

To include finite ion temperature a kinetic treatment that allows to describe the most energetic ions has to be considered :

$$
\begin{gathered}
\frac{\partial f}{\partial t}+v \frac{\partial f}{\partial x}-e \frac{\partial \phi}{\partial x} \frac{\partial f}{\partial v}=0, \\
e \phi=e \phi_{p}+e \phi_{t h}=\cos x+\mu n_{e}^{2}, \\
n_{i}=\int f d v, \\
\frac{\partial^{2} \phi}{\partial x^{2}}=\nu\left(n_{e}-n_{i}\right) .
\end{gathered}
$$

Finite ion temperature will give rise to two different effects. First of all, the maximum attainable amplitude of the grating will be reduced. Indeed the study of a nonlinear ion acoustic wave with large-amplitude sinusoidal velocity perturbation and no driver [37] with the waterbag model results in a limiting value of the density inversely proportional to the initial ion temperature $n_{\max } \simeq 6^{1 / 3}\left(v_{m} / \delta v\right)^{2 / 3} n_{0}$. An analogous effect will be present in our case. By using the estimate above we observe that the normalized thermal potential is reduced : $e \phi_{t h}=\mu n_{\max }^{2} \propto T_{e 0} / T_{i 0}^{2 / 3}$. The different regimes identified in the cold ion case will still exist: reflection, transition and crossing, but the thresholds between these three regimes will imply different values of the $\mu$ parameter.

When the ion temperature is high enough a second effet is expected. Namely a steady state solution with a population of trapped ions. To understand this effect let us neglect the thermal potential and the deviation between ion and electron density, then $\operatorname{Eq}(6)$ becomes simply :

$$
\frac{\partial f}{\partial t}+v \frac{\partial f}{\partial x}+\sin x \frac{\partial f}{\partial v}=0
$$

which describes the ions' dynamic driving by sinusoidal ponderomotive potential and is the analogous of the superradiant regime for electron waves with finite initial electron temperature[38].

This equation was studied for an initial "water-bag" velocity distribution and a static ponderomotive potential in the Hamiltonian mean-field model[39] and it was shown that the initial distribution evolves into a steadystate structure with trapped particles, characterised by the energy $\varepsilon_{h}: f(\varepsilon)=\eta_{0}\left[\Theta\left(\varepsilon_{F}-\varepsilon\right)+\chi \Theta\left(\varepsilon_{h}-\varepsilon\right) \Theta\left(\varepsilon-\varepsilon_{F}\right)\right.$. Here $\varepsilon$ is the particle energy, $\varepsilon_{F}$ the initial maximum particle energy in the water-bag model, and $\eta_{0}$ and $\chi$ parameters that depend on the fraction of trapped particles. We can expect a similar evolution in our system for a large ion temperature and an initially gaussian distribution function, leading to static plasma grating. This was also found in kinetic simulations Ref[38] for electron plasma gratings.

The trapping of the ions and the equilibrium with a steady-state ion grating implies that the average ion kinetic energy $\left(T_{i 0}\right)$ should be comparable to the maximum of the ponderomotive potential $\left(m_{e} v_{0}^{2} / 2\right)$ as seen by the ions via the electrons. In general the contribution of the electron thermal potential, even if reduced because of the ions temperature $\left(e \phi_{t h} \propto T_{e 0} / T_{i 0}^{2 / 3}\right)$ will not be negligible. In laboratory experiments, especially on short time scales, the electron temperature is usually higher than the ion temperature $T_{e 0} \geq T_{i 0}$, thus both effects will be present for different populations : ion reflection and ion trapping.

PIC simulations are conducted to show the two expected effects. The simulation parameters here are the same as those in $\operatorname{Fig}(2)$, except for the electron temperature and ion temperature. In $\operatorname{Fig}(14), T_{e 0}=T_{i 0}=$ $920 \mathrm{eV}$, which corresponds to $\mu=1.5$ and $T_{i 0}=m_{e} v_{0}^{2} / 2$. 

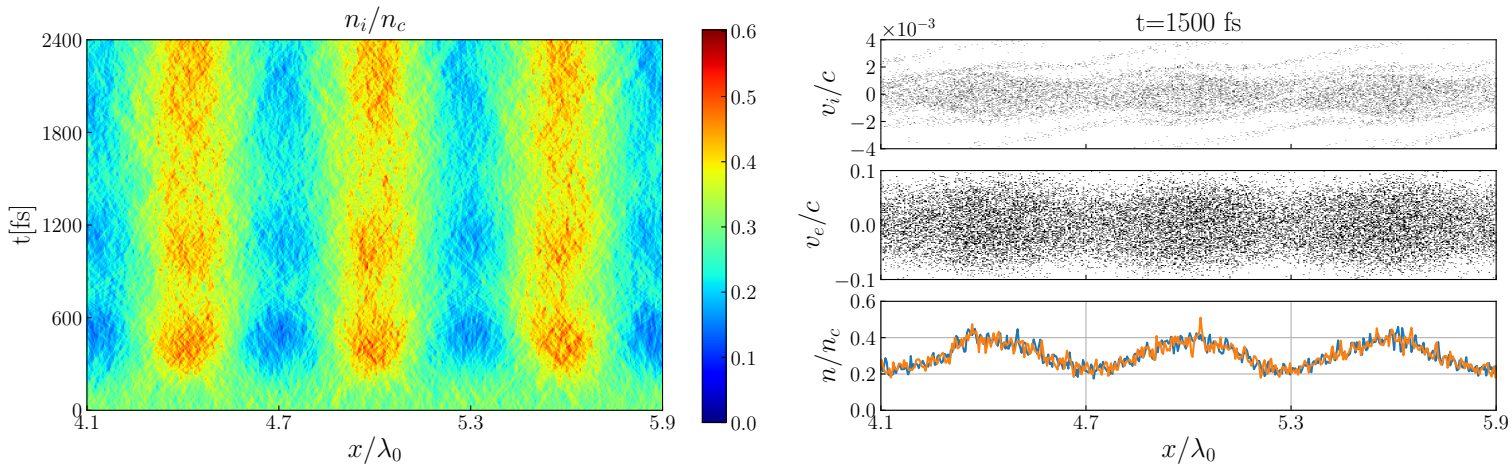

FIG. 14: Left: the amplitude of the grating(denoted by $\left.n_{i} / n_{0}\right)$ versus time. Right: from up to bottom: ion phase space, electron phase space, ion density(orange line) and electron density(blue line) at $t=1500 \mathrm{fs}$. In this simulation, $T_{e 0}=Z T_{i 0}=$ $920 \mathrm{eV}, \mathrm{Z}=1$.
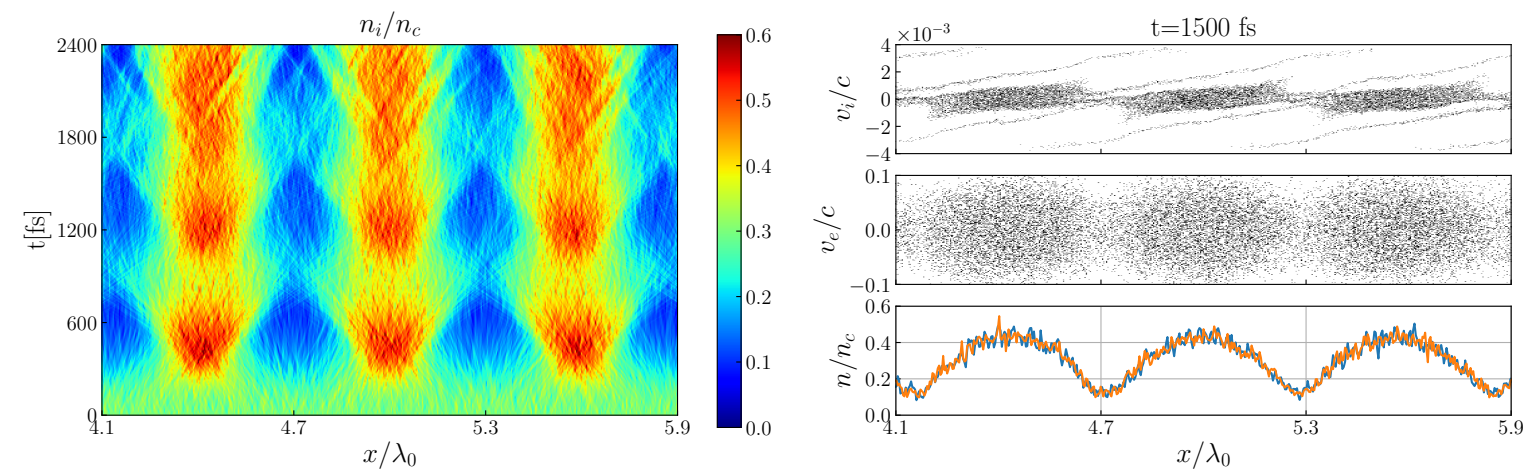

FIG. 15: The same as Fig(14), except that $T_{i 0}=200 \mathrm{eV}$.

In this case we expect mainly ion trapping a small part of the ions is reflected. Indeed compared with $\operatorname{Fig}(2)$, the grating amplitude is smaller and a quasi-static plasma grating appears. The time evolution of the phase space (not shown here) confirms that there is a population of trapped ions in the potential well that conserves the structure shown in the top panel on the right in Fig(14). By keeping the same value of $\mu$ and decreasing only the ion temperature, $T_{i 0}=200 \mathrm{eV}$, so that $T_{i 0}<m_{e} v_{0}^{2} / 2$, we do not obtain anymore a steady state solution. As shown in Fig(15) the grating amplitude is higher than in Fig(14), but less than that of the case with negligible $T_{i 0}$, and it oscillates in time. The population of ions trapped in the potential well is not present anymore.

If we maintain this value of the ion temperature but the electron temperature is decreased as well, i.e. for smaller values of $\mu$, we expect only a reduction of the grating amplitude if compared with a case with the same value of $\mu$ and completely negligible ion temperature, but no steady state solution. Simulations (not shown here) with $T_{e 0}=T_{i 0}=200 \mathrm{eV}$ show indeed this behaviour: an oscillating grating is formed with maximum values of the amplitude larger than in Fig(15) but reduced with respect to the case of smaller $T_{i 0}$.

\section{B. Consideration of particle collisions}
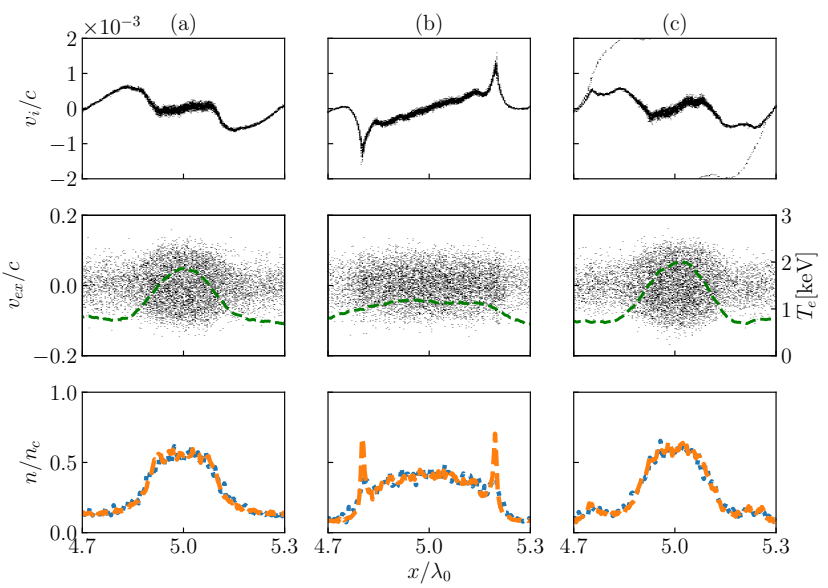

FIG. 16: PIC simulation with the same parameters as Fig(2), except that electron-ion collisions are included. Times shown are (a) $t=500 \mathrm{fs},(\mathrm{b}) t=800 \mathrm{fs},(\mathrm{c}) t=1300 \mathrm{fs}$.

The electron-ion and electron-electron collision fre- 
quency increase with increasing density and decreasing electron temperature to the power $3 / 2$ times a function weakly dependent on density and temperature, the socalled Coulomb logarithm $\ln \Lambda[40,41]$.

During the adiabatic compression the temperature increases and the collision frequency will thus decrease as the compression takes place and the grating forms. For example, for $\operatorname{Fig}(2)$, at the beginning when the plasma is not perturbed, $\ln \Lambda \approx 7, \nu_{e i} \approx 2.48 \times 10^{11} / \mathrm{s}$. When the plasma grating reaches its maximum amplitude, i.e. at time $t=500 \mathrm{fs}(\operatorname{Fig}(2 \mathrm{a}))$, the density in the grating increases to $n_{e}=0.55 n_{c}$, but the temperature also increases to $T_{e} \simeq 2 \mathrm{keV}$, so we have $\ln \Lambda^{\prime} \approx 7.7$, $\nu_{e i}^{\prime} \approx 1.49 \times 10^{11} / \mathrm{s}$. Both $\nu_{e i}^{-1}$ and $\nu_{e i}^{\prime}{ }^{-1}$ are on the order of several ps, much longer than the typical dynamic time of the plasma grating, so we expect they will have little effect on the plasma grating formation and evolution. The PIC simulation results are shown in Fig(16), the electron-ion collisions are included and there are no significant differences with Fig(2) except that the ion spikes at the edges of the grating are smoothed.
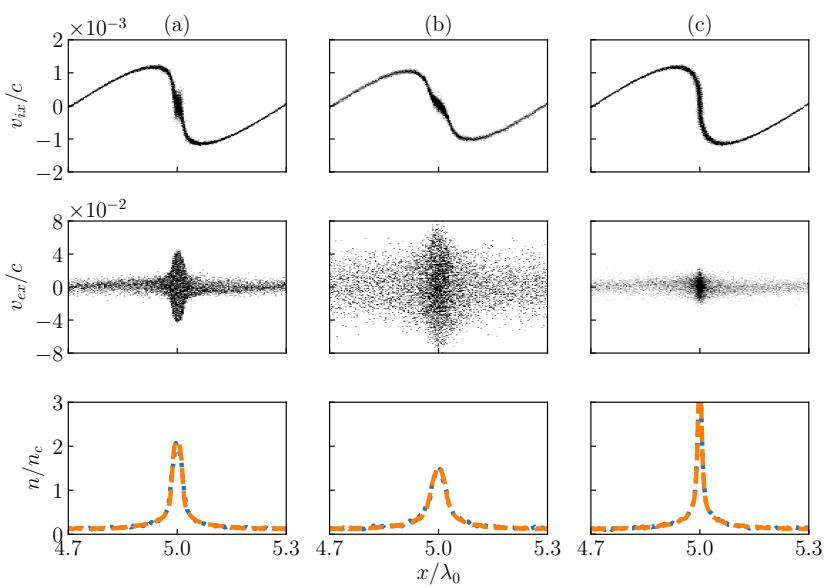

FIG. 17: PIC simulation at $t=500 \mathrm{fs}$ with the parameters same as $\operatorname{Fig}(9)$, i.e. $\mu=0.02$, except (a) is without collisions, (b) is with all the collisions(electron-ion,electron-electron,ionion), (c) is only with electron-electron collision.

The situation is different when the initial electron temperature is very low, e.g. for $\operatorname{Fig}(9), T_{e}=12.3 \mathrm{eV}, \mu=$ 0.02. In that case we have $\ln \Lambda \approx 3, \nu_{e i} \approx 6.88 \times 10^{13} / \mathrm{s}$, $\nu_{e i}^{-1} \approx 14.5 \mathrm{fs}$. In this case, the electrons are heated via inverse bremsstrahlung by the electromagnetic wave through the electron-ion collisions[40], since $\nu_{e i} t_{u n i t} \gg 1$. The overall temperature increased is higher than that of the case only considering the adiabatic heating, it results in a slightly reduced peak value of the grating and longer lifetime. This is shown in Fig(17): when the collisions are not considered in Fig(17a), the grating dynamic is in the transition regime and the peak value of the grating is twice $n_{c}$. When collisions are included Fig(17b), the electron spread in the phase space is wider than in the previous case, and the electrons are heated by electron- ion collisions. As a consequence the grating maximum amplitude is slightly smaller and the time evolution of the grating dynamic increases, even if it is still in the transition regime. The typical ion crossing time becomes about $1750 \mathrm{fs}$ instead of $800 \mathrm{fs}$ without collisions(not shown).

The e-e collision frequency is of the same order of the e-i collisions, $\nu_{e e}=\nu_{e i} / Z$ and $Z=1$ here. These collisions will induce energy transfer among the electrons during the adiabatic compression, reducing the adiabatic temperature increase. This effect is shown by artificially switching-off of the e-i collisions. The result is shown in $\operatorname{Fig}(17 \mathrm{c})$.

To summarize, the electron-ion collisions leads to additional heating of the electrons besides the adiabatic heating, but whether the collisions are important or not depends on the initial state. If in the unperturbed plasma the reciprocal of the collision frequency is already on the same order of or even higher than the dynamic time of the grating it will have a strong effect on the grating dynamic. In the opposite case in which the collision time is longer than the dynamic time of the grating, the effect of collisions can be neglected.

Moreover we wish to point out that the model we propose is not limited to lasers in the visible domain interacting with under-dense plasmas. It can be applied to X-ray laser-generated plasma grating, laser-generated plasma grating on solid surface, and infrared-laser-generated plasma grating. In the two former cases, the collisions are often important, in the last one they are negligible.

\section{CONCLUSIONS}

The laser-generated plasma grating induced by strongly coupled stimulated Brillouin scattering is characterized by large-amplitude and long-lasting ion density modulation[31], which can last much longer than the electron grating[38, 42]. Combined with its high damage threshold, this kind of plasma grating is useful to manipulate ultraintense and ultrashort lasers. Many important applications are proposed theoretically. To experimentally realise these applications, it is necessary to understand the mechanism of the formation, collapse process and how experimental parameters affect the properties of the plasma grating.

In this paper, a fluid model and adiabatic compression assumption is proposed to explain the nonlinear dynamics of plasma grating. Good agreements are found with the corresponding PIC simulation in specific cases, which validate the fluid model and adiabatic compression assumption. Proofs of the adiabatic heating are also found in PIC simulation. Further simulation with different $\mu$ in both fluid and PIC shows that as $\mu$ decreases, maximum grating amplitude is higher and the minimum grating width is smaller. Three different regimes of dynamics of the plasma grating are also found: complete reflection, transition regime and crossing. The complex dynamics of the grating in the transition regime are discussed in 
details. Analytical solutions of the simplified model are also presented, which help explain the grating's behavior. The parameter window on how $\mu$ depends on laser intensity and electron temperature is also shown, which facilitates choosing suitable experiment parameters to obtain plasma grating with desired properties. The finite ion temperature is found to have two effects on the grating dynamics. First, as the ion temperature increases, the attainable grating amplitude is smaller. Second, when the ion kinetic energy is comparable to the ponderomotive potential, the ions will be trapped steadily in the potential. One may think that the localization of particles in the grating can lead to severe collisions, which can not be ignored in our model. But we show that as the grating forms, the adiabatic compression heats the electron besides localizing the ions, which mitigates the collisions. Whether the collisions need to be considered or not depends on the initial state of the plasma.

This model also helps to explain the formation of plasma density modulation in some special cases. For example, this may be able to explain the periodic density grating driven by the beating of laser projected on the surface of a solid target and the reflected laser[28]. The periodic formation and collapse of grating has been found in the case of high-intensity laser interacting with plasma $[34,43]$, which can explain the periodic changes of the reflectivity of SBS in inertial confinement fusion.

This work is focused on the mechanism of the grating dynamics and one-dimensional situation. In the case of small-scale plasma and long laser pulses, in which the effects of energy transfer between lasers and the laser intensity distribution can be neglected, our model provides important instructions for practically building a plasma grating device. But in real experiments, these approximations usually do not hold, then it is necessary to further investigate how the energy transfer between lasers and the laser distribution in 3-dimensional space affect the grating formation.

\section{ACKNOWLEDGEMENTS}

The authors wish to acknowledge useful discussions with F. Amiranoff and J. Wurtele. H.P. acknowledges the discussions with $\mathrm{R}$. Li. This work has been done within the LABEX Plas@par project, and received financial state aid managed by the Agence Nationale de la Recherche, as part of the program "Investissements d'avenir" under the reference ANR-11-IDEX-000402. H.P acknowledges the funding from China Scholarship Council, and is partially founded by the Natural Science Foundation of China and 11875240 and Key lab foundation of CAEP 6142A0403010417. S.W. was supported by the project Advanced research using high intensity laser produced photons and particles (ADONIS) (CZ.02.1.01/0.0/0.0/16_019/0000789) and by the project High Field Initiative (HiFI) (CZ.02.1.01/0.0/0.0/15_003/0000449), both from Euro- pean Regional Development Fund. The results of the Project LQ1606 were obtained with the financial support of the Ministry of Education, Youth and Sports as part of targeted support from the National Programme of Sustainability II (SW).

\section{APPENDIX A: REFLECTION OF PUMP LASERS BY ESTABLISHED PLASMA GRATING}

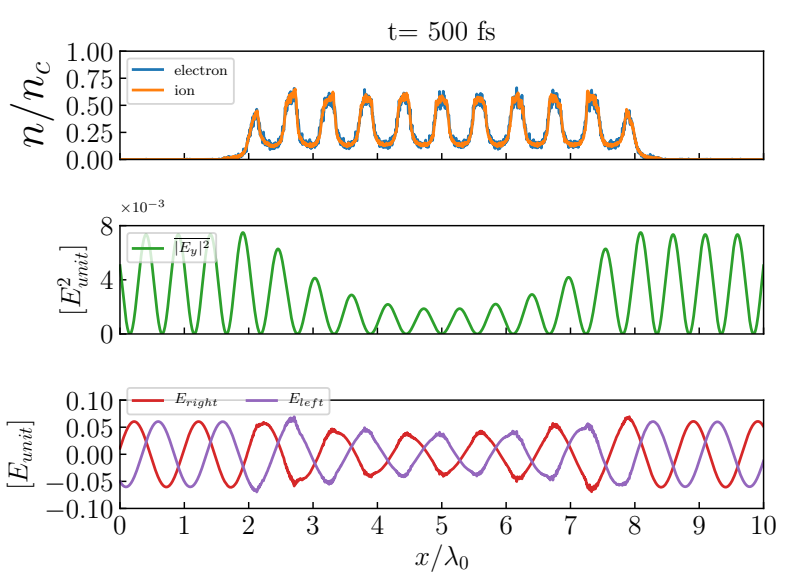

FIG. 18: From up to bottom are the plasma densities, mean squared transverse electrical field, transverse electrical field transporting to the right and the left at $t=500 \mathrm{fs}$, respectively.

Data from PIC simulation of Fig(2) is analysed. The results at $t=500 \mathrm{fs}$ are shown in Fig(18), in the first row one finds that the plasma grating has been fully established at this time.

The second row shows the ponderomotive potential characterized by the mean squared transverse electrical field $\overline{\left|E_{y}\right|^{2}}$. Note that in the free space,

$$
\begin{aligned}
\left|E_{y}\right|^{2} & =\left|E_{y 1}+E_{y 2}\right|^{2} \\
& =\left|E_{0} \cos (k x-\omega t)+E_{0} \cos (-k x-\omega t+\Delta \phi)\right|^{2} \\
& =E_{0}^{2} \cos ^{2}(k x-\omega t)+E_{0}^{2} \cos ^{2}(-k x-\omega t+\Delta \phi) \\
& +E_{0}^{2}[\cos (-2 \omega t+\Delta \phi)+\cos (2 k x-\Delta \phi)],
\end{aligned}
$$

where $E_{y 1}$ and $E_{y 2}$ are the transverse electrical fields of two pump lasers, $E_{0}$ is their amplitude, $\omega$ and $k$ are the pump frequency and wavevector, $\Delta \phi$ is their phase discrepancy, respectively. After averaged in one optical cycle, i.e. $T=2 \pi / \omega$ :

$$
\overline{\left|E_{y}\right|^{2}}=E_{0}^{2}[1+\cos (2 k x-\Delta \phi)] .
$$

It is well known the spatial derivative along $\mathrm{x}$ of $\overline{\left|E_{y}\right|^{2}}$ is the ponderomotive force generated by the beating of two pump lasers. $\overline{\left|E_{y}\right|^{2}}$ is shown in normalized unit. $E_{\text {unit }}=m_{e} c \omega / e$, for pump lasers with $a_{0}=0.06$, 
$E_{0}=0.06 E_{\text {unit }}$. So for laser without reflection, $\overline{\left|E_{y}\right|^{2}}$ should range from 0 to $7.2 e-3$. While in the case the grating is fully established as shown in $\operatorname{Fig}(18), \overline{\left|E_{y}\right|^{2}}$ is smaller in the center which should be explained by that the grating reflects the following injected lasers, the lasers' transmittance drops as the lasers get closer to the center. If with enough layers of plasma grating, one could see the ponderomotive potential drops to zero in the center.

The third row shows the lasers propagating to each direction: $E_{\text {right }}$ and $E_{\text {left }}$, note that

$$
\begin{aligned}
E_{\text {right }} & =\left(E_{y}+\frac{v_{p}}{c} B_{z}\right) / 2, \\
E_{\text {left }} & =\left(E_{y}-\frac{v_{p}}{c} B_{z}\right) / 2, \\
v_{p} & =\frac{c}{\sqrt{1-n_{e} / n_{c}}},
\end{aligned}
$$

where $v_{p}$ is the phase velocity of the lasers, $n_{e}$ is the local electron density. $E_{\text {right }}$ and $E_{\text {left }}$ are attenuated by the grating because of the reflection in Fig(18).

It is proven here that the ponderomotive potential actually decreases in the grating when the grating is fully established because the grating reflects the pump lasers. The ponderomotive restores to it's original level after the grating collapses. This effect is not included in Sec(??), it partly attributes to the differences between the fluid model and the PIC simulation. But the PIC simulations already supports the model well, and give enough information of the grating dynamics. While for the further study of plasma grating dynamics in a large-scale plasma, both the energy exchange between pump lasers and the reflection by the grating should be considered.

\section{APPENDIX B: ANALYTICAL SOLUTION OF THE FLUID MODEL}

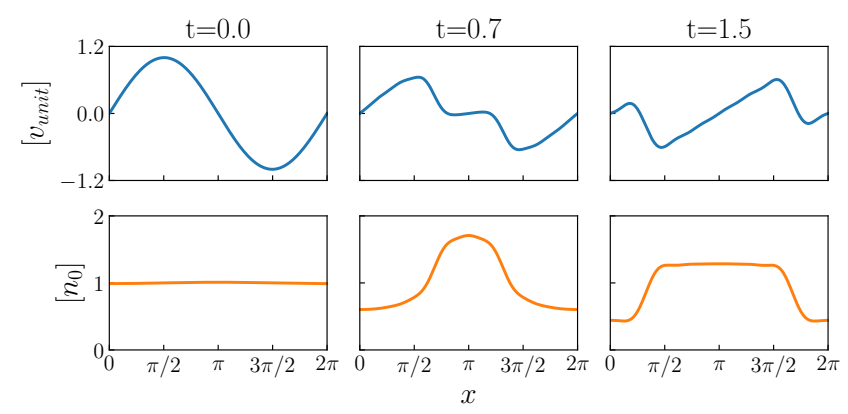

FIG. 19: The ion velocity and ion density at different times when $\mu=1.0$ according to the analytical solutions.

In Section(III), we have shown that $\nu$ has little effect on the main results as long as $\nu \gg 1$. So in the model function $\operatorname{Eq}(5), \operatorname{Eq}(5 \mathrm{a})$ can be neglected and $n_{e}$ can be replaced with $n_{i}$ in $\operatorname{Eq}(5 \mathrm{c})$. The model is still strongly nonlinear and with a spatially inhomogeneous term $\sin x$, one can simplify the model and the solution by assuming that the velocity has an initial sinusoidal distribution and no spatially driving term. Then the model is simplified to:

$$
\begin{gathered}
\frac{\partial n_{i}}{\partial t}+\frac{\partial\left(n_{i} v_{i}\right)}{\partial x}=0 \\
\frac{\partial v_{i}}{\partial t}+v_{i} \frac{\partial v_{i}}{\partial x}+\mu \frac{\partial n_{i}^{2}}{\partial x}=0
\end{gathered}
$$

with the initial conditions: $\left.v_{i}\right|_{t=0}=\sin x$ and $\left.n_{i}\right|_{t=0}=1$. Then multiply $\mathrm{Eq}(\mathrm{B} 1 \mathrm{a})$ with $\pm \sqrt{2 \mu}$ and add to $\mathrm{Eq}(\mathrm{B} 1 \mathrm{~b})$, we obtain:

$$
\frac{\partial u}{\partial t}+u \frac{\partial u}{\partial x}=0
$$

with $u=v_{i} \pm \sqrt{2 \mu} n_{i}$ and the initial condition $\left.u\right|_{t=0}=$ $\sin x \pm \sqrt{2 \mu}$. Transforming from Eulerian variables $(x, t)$ to Lagrangian variables $\left(x_{0}, \tau\right)$ :

$$
\tau=t, \quad x_{0}=x-\xi, \quad \xi=\int_{0}^{\tau} u\left(x_{0}, \tau^{\prime}\right) d \tau^{\prime},
$$

$\mathrm{Eq}(\mathrm{B} 2 \mathrm{a})$ is just:

$$
\frac{\partial u}{\partial \tau}=0
$$

Its solution is $u\left(x_{0}, \tau\right)=u\left(x_{0}, 0\right)=\sin x_{0} \pm \sqrt{2 \mu}$. Then the spatial variable transform is $x=x_{0}+\tau\left(\sin x_{0} \pm \sqrt{2 \mu}\right)$, or $x \mp \sqrt{2 \mu} \tau=x_{0}+\tau \sin x_{0}$. We then know that $\sin x_{0}$ is a periodic function of $x \mp \sqrt{2 \mu} \tau$ with period $2 \pi$, and it can be transformed back to Eulerian variables with the following Fourier-series[44]:

$$
\begin{gathered}
\sin x_{0}=\sum_{n=1}^{\infty} a_{n}(t) \sin n(x \mp \sqrt{2 \mu} t), \\
a_{n}(t)=(-1)^{n+1} \frac{2}{n t} J_{n}(n t),
\end{gathered}
$$

where $J_{n}$ is the $n$th Bessel function of the first kind. Then $u$ has two solutions: $u=u_{1,2}= \pm \sqrt{2 \mu}+$ $\sum_{n=1}^{\infty} a_{n}(t) \sin n(x \mp \sqrt{2 \mu} t)$ and:

$$
\begin{array}{r}
v_{i}=\frac{u_{1}+u_{2}}{2}=\sum_{n=1}^{\infty} b_{n}(t) \sin n x, \\
b_{n}(t)=(-1)^{n+1} \frac{2 \cos \sqrt{2 \mu} n t}{n t} J_{n}(n t), \\
n_{i}=\frac{u_{1}-u_{2}}{2 \sqrt{2 \mu}}=1+\sum_{n=1}^{\infty} c_{n}(t) \cos n x, \\
c_{n}(t)=(-1)^{n} \frac{2 \sin \sqrt{2 \mu} n t}{\sqrt{2 \mu} n t} J_{n}(n t) .
\end{array}
$$

When $\mu=0$, the solutions converge to the cold limit, we have: $b_{n}(t)=(-1)^{n+1}(2 / n t) J_{n}(n t), c_{n}(t)=$ 
$(-1)^{n} 2 J_{n}(n t)$, and in the center of the grating center(at $x=\pi) n_{i}=1 /(1-t), \partial v_{i} / \partial x=-t /(1-t)$, noting that $\cos (n \pi)=(-1)^{n}$ and $\sum_{n=1}^{\infty} J_{n}(n t)=t / 2(1-t)$. This means that as the ions keep accumulating to the grating $\operatorname{center}(x=\pi)$ and time goes from $t=0$ to $t=1$, the grating density goes to infinity and the slope of the ion velocity goes to minus infinity. At $t=1$ the grating finally breaks due to that the ion sheets cross each other.

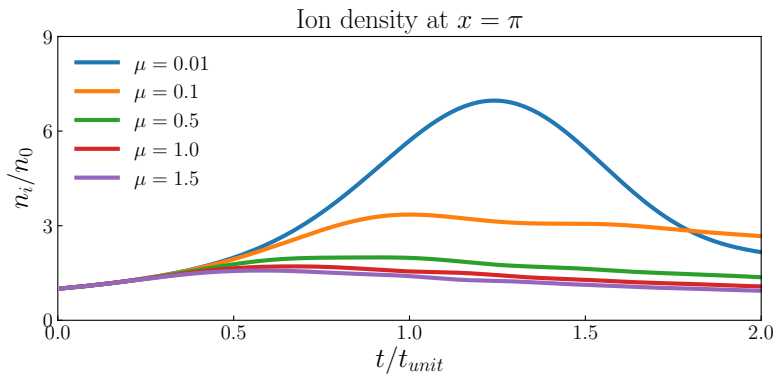

FIG. 20: In the grating center, the ion density evolution in time with different $\mu$ according to the analytical solutions.

When $\mu>0$, the results given above are quite complex to analyses. But one can cut the infinite series to the first several terms which gives very good approximations, and the numerical results can be provided readily by scientific softwares, e.g. Mathmatica. Fig(19) shows the ion velocity and density at different times with $\mu=1.0$ as given by $\mathrm{Eq}(\mathrm{B} 6)$ cut at $n=10$. One can see clearly the formation of velocity plateau and density grating at $t=0.7$, the ion reflection and grating stretch at $t=1.5$. Fig $(20)$ shows the temporal evolution of the maximum grating amplitude along $x$ with different $\mu$ by cutting $\mathrm{Eq}(\mathrm{B} 6 \mathrm{c})$ at $n=10$. At finite $\mu$, the ion density doesn't go to infinity, and as $\mu$ gets bigger then peak grating amplitude decreases, which agrees well with the simulations in SectionIII.For the limit that $\mu$ is very big, $\mathrm{Eq}(\mathrm{B} 1)$ can be linearized with $n_{i}=1+\tilde{n}, v_{i}=\tilde{v}$ and has solutions: $n_{i}=1-\sin (x-\sqrt{2 \mu} t) / \sqrt{2 \mu}, v_{i}=\sin (x-\sqrt{2 \mu} t)$. The velocity and density perturbation are quite small and linear.

In this appendix, the analytical solutions of homogeneous fluid model(no driving ponderomotive potential but the ion velocity has an initial sinusoidal perturbation) are found, which supports the simulation results in previous sections. They can also be used to predict the grating behavior conveniently.
[1] M. Perry et al., Optics letters 24, 160 (1999).

[2] C. Danson et al., Nuclear Fusion 44, S239 (2004).

[3] E. W. Gaul et al., Design of the texas petawatt laser, in Conference on Lasers and Electro-Optics, page JFB2, Optical Society of America, 2005.

[4] L. Yu et al., Optics express 26, 2625 (2018).

[5] F. Lureau et al., 10 petawatt lasers for extreme light applications, in Solid State Lasers XXIX: Technology and Devices, volume 11259, page 112591J, International Society for Optics and Photonics, 2020.

[6] O. Uteza et al., Applied Surface Science 254, 799 (2007).

[7] K. R. Kafka et al., Optics express 24, 28858 (2016).

[8] Q. Meng, J. Yu, Z. Zhong, R. Ye, and B. Zhang, Optical Materials 46, 444 (2015).

[9] A. Ollé, S. Diop, N. Roquin, L. Gallais, and L. Lamaignère, Optics Letters 45, 4024 (2020).

[10] C. Thaury et al., Nature Physics 3, 424 (2007).

[11] H. Peng et al., Matter and Radiation at Extremes 4, 065401 (2019).

[12] J.-R. Marquès et al., Physical Review X 9, 021008 (2019).

[13] R. Kirkwood et al., Nature Physics 14, 80 (2018).

[14] M. Nakatsutsumi et al., Optics letters 35, 2314 (2010).

[15] A. Leblanc et al., Nature Physics 13, 440 (2017).

[16] P. Michel, L. Divol, D. Turnbull, and J. D. Moody, Physical Review Letters 113, 205001 (2014).

[17] D. Turnbull et al., Physical Review Letters 116, 205001 (2016).

[18] D. Turnbull et al., Physical Review Letters 118, 015001 (2017).

[19] G. Lehmann and K.-H. Spatschek, Physical review letters 116, 225002 (2016).

[20] H.-C. Wu, Z.-M. Sheng, Q.-J. Zhang, Y. Cang, and J. Zhang, Physics of plasmas 12, 113103 (2005).
[21] H.-C. Wu, Z.-M. Sheng, and J. Zhang, Applied Physics Letters 87, 201502 (2005).

[22] G. Lehmann and K. Spatschek, Physical Review E 97, 063201 (2018).

[23] A. Leblanc et al., Nature Physics 13, 440 (2017).

[24] G. Lehmann and K. Spatschek, Physical Review E 100, 033205 (2019).

[25] S. Monchoce et al., Phys Rev Lett 112, 145008 (2014).

[26] D. Forslund, J. Kindel, and E. Lindman, The Physics of Fluids 18, 1002 (1975).

[27] D. Forslund, J. Kindel, and E. Lindman, Physics of Fluids 18, 1017 (1975).

[28] A. Andreev, C. Riconda, V. Tikhonchuk, and S. Weber, Physics of plasmas 13, 053110 (2006).

[29] D. Forslund, J. Kindel, K. Lee, and B. Godfrey, Physics of Fluids 22, 462 (1979).

[30] Z.-M. Sheng, J. Zhang, and D. Umstadter, Applied Physics B 77, 673 (2003).

[31] H. Peng, C. Riconda, M. Grech, J.-Q. Su, and S. Weber, Physical Review E 100, 061201 (2019).

[32] G. Shvets, N. J. Fisch, A. Pukhov, and J. Meyer-ter Vehn, Physical review letters 81, 4879 (1998).

[33] M. Chiaramello et al., Physics of Plasmas 23, 072103 (2016).

[34] S. Weber, C. Riconda, and V. Tikhonchuk, Physical review letters 94, 055005 (2005).

[35] J. Derouillat et al., Computer Physics Communications 222, 351 (2018).

[36] G. Lehmann and K. Spatschek, Physics of Plasmas 24, 056701 (2017)

[37] H. Ikezi and P. Folkes, The Physics of Fluids 23, 314 (1980).

[38] G. Lehmann and K. Spatschek, Physics of Plasmas 26, 
013106 (2019).

[39] R. Pakter and Y. Levin, Physical review letters 106, 200603 (2011)

[40] W. L. Kruer and J. M. Dawson, The Physics of Laser Plasma Interactions, 1988

[41] J. Huba, NRL PLASMA FORMULARY, 2009.
[42] H. Chen et al., Physics of Plasmas 17, 083112 (2010).

[43] S. Weber, C. Riconda, and V. Tikhonchuk, Physics of plasmas 12, 043101 (2005).

[44] R. C. Davidson and J. E. Scherer, IEEE Transactions on Plasma Science 1, 58 (1973). 